\title{
Kondo Insulator description of spin state transition in $\mathrm{FeSb}_{2}$
}

\author{
${ }^{\ddagger}$ C. Petrovic, ${ }^{\ddagger} \S$ Y. Lee, ${ }^{\ddagger} \S$ T. Vogt, ${ }^{\boldsymbol{\top}} \mathrm{N}$. Dj. Lazarov, ${ }^{\star}$ S. L. Bud’ko and ${ }^{\star} \mathrm{P}$. C. Canfield \\ ${ }^{\ddagger}$ Physics Department, Brookhaven National Laboratory, Upton, New York 11973-5000 \\ $\S$ Center for Functional Nanomaterials, Brookhaven National Laboratory, Upton, New York 11973-5000 \\ Laboratory of Theoretical and Solid State Physics, \\ Institute of Nuclear Sciences - Vinca, P. O. Box 522, 11001 Belgrade, Serbia \\ * Ames Laboratory and Department of Physics and Astronomy, Iowa State University, Ames, Iowa 50011
}

(Dated: July 6, 2021)

\begin{abstract}
The thermal expansion and heat capacity of $\mathrm{FeSb}_{2}$ at ambient pressure agrees with a picture of a temperature induced spin state transition within the $\mathrm{Fe}_{2 g}$ multiplet. However, high pressure powder diffraction data show no sign of a structural phase transition up to 7GPa. A bulk modulus $\mathrm{B}=84(3) \mathrm{GPa}$ has been extracted and the temperature dependence of the Grüneisen parameter has been determined. We discuss here the relevance of a Kondo insulator description for this material.
\end{abstract}

PACS numbers: $75.30 . \mathrm{Mb}, 71.28 .+\mathrm{d}, 75.20 . \mathrm{Hr}$

$\mathrm{FeSb}_{2}$ is a small gap semiconductor whose magnetic properties strongly resemble FeSi. The magnetic susceptibility of FeSi evolves from a high temperature maximum to a flat van-Vleck like behavior as $\mathrm{T} \rightarrow 0$ with a Curie tail which is sample dependant and attributed to defects and impurities. Aeppli and Fisk have pointed out that the underlying physics of FeSi is similar to the physics of Kondo insulators with localized magnetic moments $\frac{1}{1}$ This picture was also supported by other measurements as well 2,3 . 3 All Kondo insulator materials are cubic with the exception of CeRhSb and CeNiSn and are all $4 f$ intermetallics, except $\mathrm{FeSi}, \mathrm{RuAl}_{2}$ and $\mathrm{Fe}_{2} \mathrm{VAl}_{2}, \frac{1}{4}, \frac{5}{\mathrm{~A}} \mathrm{~A}$ simple model that explains their properties involves a flat $\mathrm{f}$ band hybridizing with a broad conduction band with two electrons per unit cell. At $\mathrm{T}=0$ the electrons populate a lower hybridized band, and with the increase of $\mathrm{T}$ the electrons start populating the higher band, resulting in a thermally activated Pauli susceptibility. The Anderson Kondo lattice model with two electrons per site with one $f$ and one conduction band thus captures the important physics. Cubic structures are more favorable for this scenario since the requirement of just one band crossing the Fermi level is more likely.

Another way to think about FeSi is based on the picture of a nearly magnetic semiconductor in an itinerant model $\underline{6}$. approximation (LDA) band structure calculations ${ }^{\frac{8}{9} 9}$ An important question to answer is if the itinerant picture is correct or if the framework of the Anderson model with a reduced on-site Coulomb repulsion $U$ better describes the physics of $\mathrm{FeSi}$. Other possible model systems containing $3 d$ metals where these scenarios can be studied are therefore highly sought after.

The small gap semiconductor $\mathrm{FeSb}_{2}$ crystallizes in the marcasite-type $\mathrm{FeS}_{2}$ structure (Fig. 1(a)) $\stackrel{10}{\underline{0}}$ The basic structural unit is made out of Fe ions surrounded by a deformed $\mathrm{Sb}$ octahedra $\stackrel{11}{1}$ The Sb octahedra share edges along the $c$ axis (Fig 1(b)). According to "classical" ligand field theory, the $\mathrm{Fe}_{2 g}$ orbitals are further split into two lower lying $\Lambda$ orbitals with $\mathrm{d}_{y z}$ and $\mathrm{d}_{x z}$ symmetries and higher lying $\Xi$ orbitals with $\mathrm{d}_{x y}$ symmetry, which are directed toward near-neighbor cations along the $c$-axis of the crystal 12 One type of marcasite the so-called "anomalous marcasite" phase is formed with cations having $\mathrm{d}^{n}$ configurations for $\mathrm{n} \geq 6$ and a large $c / a$ ratio of $0.73-0.75$ in which the $\mathrm{d}_{x y}$ orbitals are doubly occupied. The other so-called "regular marcasite" structure is formed with $\mathrm{n} \leq 4$ for smaller $c / a$ ratios of $0.53-0.57$ because the $\mathrm{d}_{x y}$ orbitals are empty $\underline{\underline{11}-13}$ Consequently, the Fe-Sb-Fe bond angle $\alpha$ between the neighboring cations in edge-sharing octahedra along the c-axis is $\alpha<90^{\circ}$ in regular and $\alpha>90^{\circ}$ in anomalous marcasites (see Fig. 1(b)) 12 Thus, if the $d^{n}$ configuration of transition metal atom has $n>4$, then it implies electrons in $\Xi$ orbitals. Repulsive forces along the c-axis will consequently increase the angle $\alpha$. For example, $\mathrm{FeS}_{2}$ with a $d^{6}$ configuration has $\alpha=97.5^{\circ}$ as opposed to $\mathrm{FeAs}_{2}$ with a $d^{4}$ configuration and $\alpha=72.5^{\circ}$. Therefore the angle $\alpha$ should increase monotonically with the number of $\Xi$ electrons from a $d^{4}$ configuration ( $\Xi$ empty) to a $d^{6}$ configuration ( $\Xi$ filled) $\underline{\underline{12}}$

The ionic configuration of iron in $\mathrm{FeSb}_{2}$ is $\mathrm{d}^{4}$ and its ground state at low temperatures is the low spin state $\mathrm{Fe}^{4+}\left(\mathrm{t}_{2 g}^{4} \mathrm{e}_{g}^{0}\right)$ with the $\Lambda$ orbital filled and the $\Xi$ orbital empty. $\stackrel{12}{=}$ Indeed, at low temperature the magnetic susceptibility of $\mathrm{FeSb}_{2}$ results entirely from the core diamagnetism and shows little temperature dependence 14 Similarly to $\mathrm{FeSi}$, above $100 \mathrm{~K}$ the susceptibility is paramagnetic $\stackrel{14,15}{15}$ One possible explanation for the enhanced magnetic susceptibility is a spin state transition of the Fe ions $\frac{14}{\underline{14}}$ It is not uncommon that in transition metal alloys the crystal field energy marginally exceeds the Hund's exchange energy and therefore energies of the order of $1 \mathrm{meV}$ can produce excitations across a spin gap. What makes anomalous marcasites unusual is the existence of a band of itinerant electronic states due to the overlap of higher lying $\Xi$ orbitals along the c-axis $\frac{12}{2}$ In this work, structural, thermodynamic and magnetic studies were carried out in an effort to further elucidate the structure-property relation in this material since a spin state transition within the $3 d$ multiplet might couple to the lattice. 


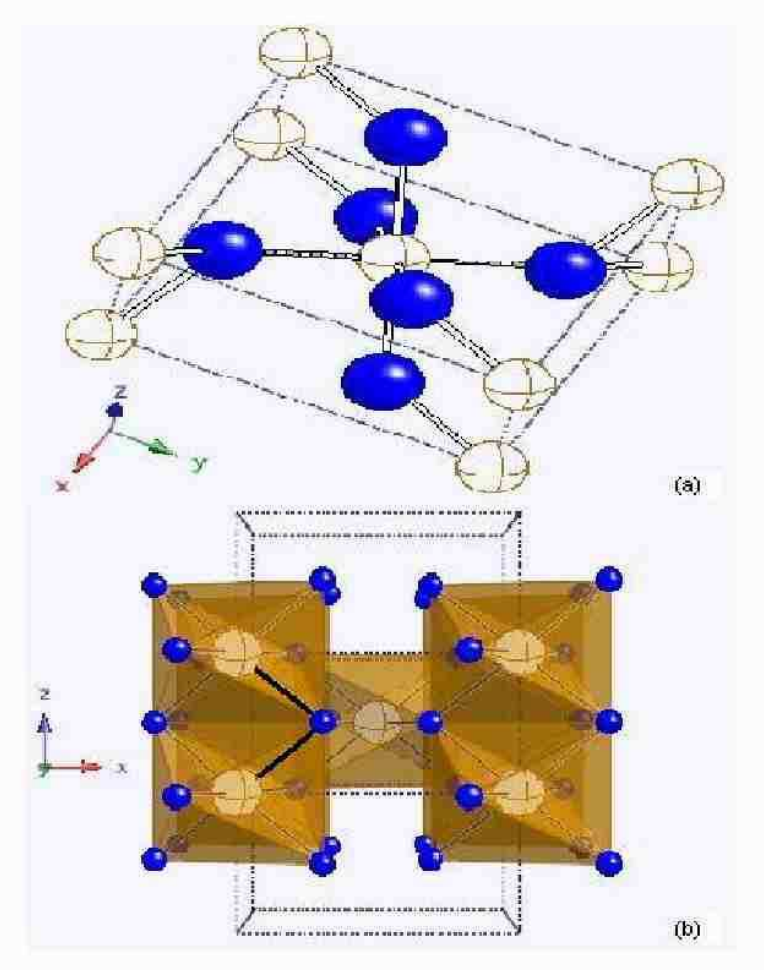

FIG. 1: (a) Crystal structure of $\mathrm{FeSb}_{2}$ showing Fe (open, brown crosses) surrounded by Sb (full blue) octahedra. The two short Fe-Sb bond distances are shown in black and white. The $\mathrm{d}_{x y}$ orbital is oriented in between short Fe-Sb bond distances along the z-axis (the c-axis of the unit cell). (b) The Fe-Sb-Fe bond angle $\alpha$ associated with the edge sharing octahedra along the c-axis of the unit cell. Note that increased occupancy of $\mathrm{d}_{x y}$ orbitals is reflected in the increase of $\alpha$.

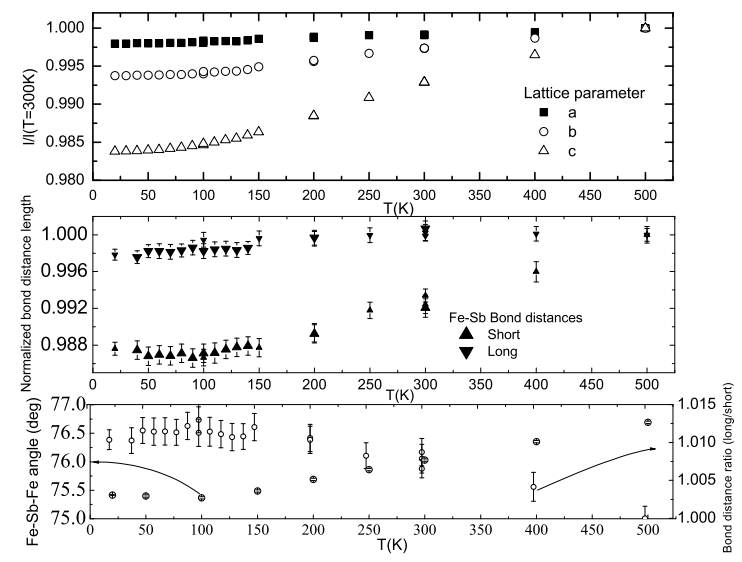

FIG. 2: Temperature dependance of (a) normalized unit cell parameters (b) Bond distance lengths and (c) Fe-Sb-Fe angle $\alpha$ between edge sharing octahedra and bond distance ratio (long/short). Note the increase of angle $\alpha$ above $\mathrm{T}_{\text {crossover }}$ (see text) and the presence of two regions of the Fe-Sb normalized short bond distance length contraction around it.
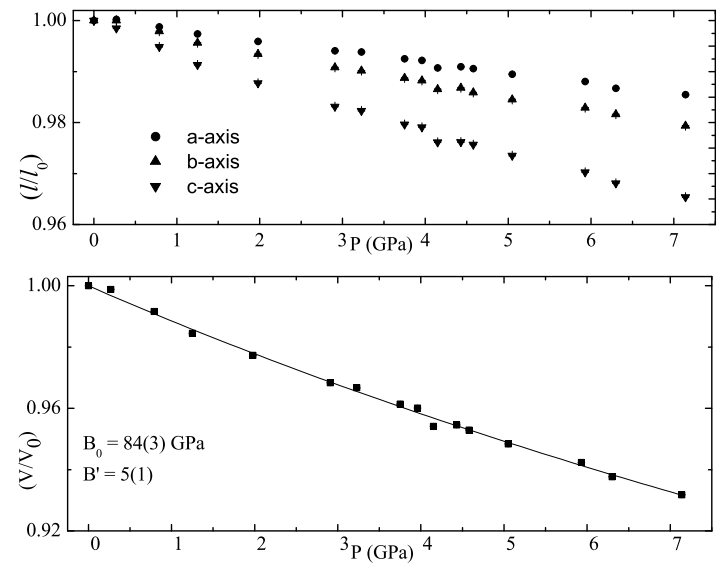

FIG. 3: Pressure dependance of (a) Normalized unit cell parameters (b) Unit cell volume of $\mathrm{FeSb}_{2}$

High quality $\mathrm{FeSb}_{2}$ single crystals have been grown from excess $\mathrm{Sb}$ flux $\stackrel{14}{\underline{n}}$ Variable temperature and high pressure experiments were performed at the beamline X7A of the National Synchrotron Light Source at the Brookhaven National Laboratory. A closed-cycle Herefrigerator and a diamond-anvil cell were used to control the sample environment, and monochromatic synchrotron X-ray and gas-proportional position-sensitive detector were used to measure the powder diffraction data $\stackrel{16}{\underline{16}}$ Magnetization and specific heat were measured in Quantum Design MPMS and PPMS instruments. Single crystals of $\mathrm{FeSb}_{2}$ were thoroughly ground to a fine powder. Rietveld refinements were performed using GSAS17. The results of fits were summarized in Tables 1 and 2 .

Fig. 2 shows the temperature evolution of several structural parameters. Linear coefficients of thermal contraction $\frac{18}{18} \alpha$ in the paramagnetic state $\frac{14}{4} \alpha_{i}=$ $1 / l(\partial l / \partial T)_{P}(\mathrm{i}=\mathrm{a}, \mathrm{b}, \mathrm{c})$ for individual unit cell axes are: $\alpha_{a}=4.2 \cdot 10^{-6} K^{-1}, \alpha_{b}=1.3 \cdot 10^{-5} K^{-1}$ and $\alpha_{c}=$ $3.4 \cdot 10^{-5} \mathrm{~K}^{-1}$. Individual $\mathrm{Fe}-\mathrm{Sb}$ bonds also change as the temperature is lowered and temperature effects on bond distance lengths normalized to $500 \mathrm{~K}$ are shown in Fig. 2(b). We observe two regions of bond contraction with a crossover near $100 \mathrm{~K}$. The thermal contraction of the long Fe-Sb bond distances (in the octahedral plane) becomes marginally stronger below 300K, whereas the short (apical) Fe-Sb bond distances contract significantly between $500 \mathrm{~K}$ and $100 \mathrm{~K}$. The contraction of the short Fe-Sb bond distances is finished at $100 \mathrm{~K}$ and below this temperature we observe no further thermal contraction on cooling down to the lowest measured temperature of $20 \mathrm{~K}$. We can estimate the bond distance contraction by calculating the mean coefficient of contraction between $500 \mathrm{~K}$ and $20 \mathrm{~K}: \bar{\alpha}_{T_{1}, T_{2}}=$ $2 /\left(i_{1}+i_{2}\right)\left[\frac{i_{1}-i_{2}}{T_{2}-T_{1}}\right]\left(\mathrm{T}_{1}=20 \mathrm{~K}, \mathrm{~T}_{2}=500 \mathrm{~K} ; \mathrm{i}=\right.$ short,long $)$ : $\bar{\alpha}_{20,500}^{\text {short }}=1.16 \cdot 10^{-5} \mathrm{~K}^{-1}, \bar{\alpha}_{20,500}^{\text {long }}=2.6 \cdot 10^{-5} \mathrm{~K}^{-1}$, in- 
dicating relatively large differences in the distortion of the octahedra. Both the bond distance ratio and the FeSb-Fe angle $\alpha$ associated with the shared octahedral-site edges along the c-axis (Fig. 2 (c)) change the slope below $100 \mathrm{~K}$. The change in the bond distance ratio implies maximum distortions for the Fe-Sb octahedra around the $\mathrm{T}_{\text {crossover }} \sim 100 \mathrm{~K}$. The value of the Fe-Sb-Fe bond angle at $300 \mathrm{~K}(76.028 \pm 0.02)^{\circ}$ agrees well with the previously reported value of $76.0^{\circ}, 19.13 \mathrm{Fig}$. 2(c) shows that the Fe$\mathrm{Sb}-\mathrm{Fe}$ bond angle $\alpha$ has little or no temperature dependance for $\mathrm{T}_{\mathrm{i}} 100 \mathrm{~K}$ and increases above $\mathrm{T}_{\text {crossover }} \sim 100 \mathrm{~K}$.

The lattice parameters contract smoothly with pressure to smaller values above $3 \mathrm{GPa}$ than the ones obtained by thermal contraction down to 20K (Fig. 3(a), Fig. 4). Individual lattice parameter compressibilities $\beta_{0}^{i}=-\frac{1}{i}\left(\frac{\partial i}{\partial P}\right)_{T}(i=a, b, c)$ are quite different: $\beta_{0}^{a}=0.00223(4) G P a^{-1}, \quad \beta_{0}^{b}=0.00339(3) G P a^{-1}$ and $\beta_{0}^{c}=0.00655(5) G P a^{-1}$. The ratio of the $c$ and $a$ axis compressibilities is as much as $\left(\frac{\beta_{c}}{\beta_{a}}\right)_{T}=2.9$. When compared with a ratio of thermal contraction $\left(\frac{\alpha_{c}}{\alpha_{a}}\right)_{P}=8$, it follows that structural changes are more anisotropic with temperature. Both temperature and pressure compress the $c$-axis which is the direction of higher lying $\Xi$ orbitals associated with $\mathrm{d}_{x y}$ symmetry rather strongly, whereas the $b$ and $a$ axis are less compressible. The ratios of contraction to compressibility are also highest for the c-axis: $\left(\frac{\alpha}{\beta}\right)^{a}=1.88 \cdot 10^{-3} \mathrm{GPaK}^{-1},\left(\frac{\alpha}{\beta}\right)^{b}=3.83 \cdot 10^{-3} \mathrm{GPaK}^{-1}$ and $\left(\frac{\alpha}{\beta}\right)^{c}=1.15 \cdot 10^{-2} G P a K^{-1}$. We see no evidence for the formation of a high pressure polymorph up to $(7.14 \pm 0.1) \mathrm{GPa}$, as was established in the cases of $\mathrm{CrSb}_{2}$ and $\mathrm{NiSb}_{2} \stackrel{20}{\underline{20}}$ The uncertainty in Rietveld refinement and the errors of the bond distances at high pressures limit our conclusions on the bond distance ratio and the Fe-SbFe octahedral angle between neighboring cations in edgesharing octahedra. However, preliminary measurements indicate that there is no change in the pressure dependence of the Fe-Sb bond distances and the angle $\alpha$ in contrast to their thermal contraction. Their ratio remains constant under pressure. Fig. 3 (b) shows the normalized unit cell volume as a function of pressure. We obtain the value of bulk modulus $\mathrm{B}_{0}=84(3) \mathrm{GPa}$ and $\mathrm{B}^{\prime}=5(1)$ by fitting a Birch-Murnagham equation of state $\underline{21}$

The Fe-Sb long and Fe-Sb short bond lengths in Fe-Sb octahedra involve $\mathrm{Fe} \mathrm{a}_{\sigma}$ bonding orbitals ( $\mathrm{e}_{g}$ in origin) and $\mathrm{Sb} \mathrm{sp}^{3}$ hybrid orbitals, as pointed out by Goodenough and Fan, ${ }^{12}, 15$ Bond distance lengths in Fe-Sb octahedra influence the large energy gap between $t_{2 g}$ and $\mathrm{e}_{g}$ derived states. The gap within the $\mathrm{t}_{2 g}$ multiplet is caused by mixing of $\Xi$ cation and $\sigma$ bonding anion orbitals, and the strength of this mixing is governed by the Fe-Sb-Fe angle $\alpha$ that measures the deviation from an ideal tetrahedral angle ${ }^{12}$ Starting from the diamagnetic state state at low temperatures, the increase of the the Fe-Sb-Fe angle $\alpha$ between neighboring cations (Fig. 1(b), Fig. 2(c)) above $\mathrm{T}_{\text {crossover }} \sim 100 \mathrm{~K}$ indicates a thermally induced population of higher lying $\Xi$ orbitals $\left(\mathrm{d}_{x y}\right.$ in ori-

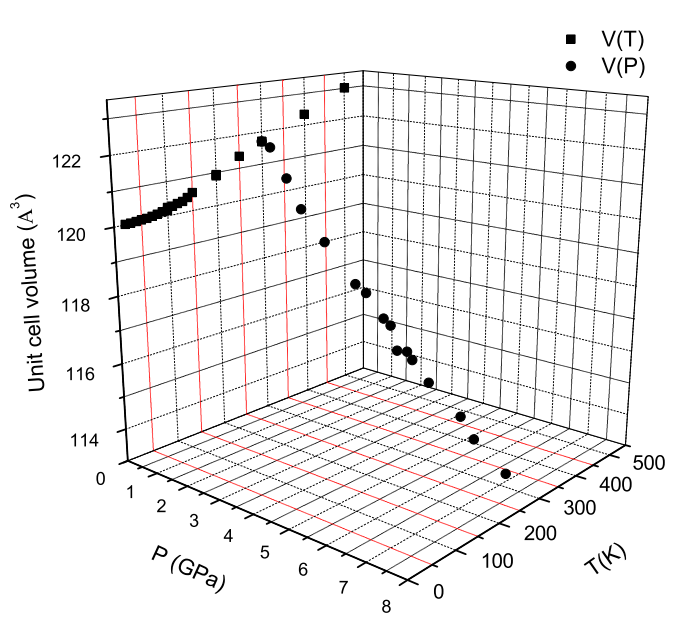

FIG. 4: The effects of pressure and temperature on the unit cell volume. The $8 \mathrm{GPa}$ of pressure reduce the unit cell volume by $1.4 \%, 4.6$ times exceeding the ambient pressure compression for $6.5 \%$ by temperature decrease from $300 \mathrm{~K}$ to $20 \mathrm{~K}$.

gin). Since $\mathrm{T}_{\text {crossover }}$ coincides with the temperature of diamagnetic to paramagnetic transition in $\mathrm{FeSb}_{2}$, we conclude that the spin state transition observed in Ref. 14 occurs within the $\mathrm{Fe}_{2 g}$ multiplet 14

Goodenough's description of the energy bands of $\mathrm{FeSb}_{2}$ can be connected with the electronic and magnetic properties of Kondo insulators. Transitions within the $\mathrm{t}_{2 g}$ multiplet involves the population of a $\mathrm{d}_{x y}$-derived band of itinerant states. In the case of a Kondo insulator we expect to describe the magnetic susceptibility with a model of a metallic spin paramagnetism, albeit with a large renormalization of the noninteracting bands. Thus, we used the model proposed by Jaccarino ${ }^{22}$ The temperature dependence of the magnetic susceptibility should be explained with a model of two narrow bands (peaks) at the density of states of width $\mathrm{W}$ and separated by $\mathrm{E}_{g}=2 \Delta$ (see inset in Fig. 5). This model has been successfully applied to show the validity of Kondo insulator picture in seminal work on the thermodynamics of $\mathrm{FeSi}^{3}, \underline{3}$

The Pauli susceptibility of an itinerant electron system with $\mathrm{N}(\mathrm{E})$ density of states is:

$$
\chi(T)=-2 \mu_{B}^{2} \int_{c-\text { band }} N(E) \frac{\partial f(E, \mu, T)}{\partial E} d E
$$

where $\mu_{B}$ is the Bohr magneton and $f(E, \mu, T)=$ $\left(\exp \left[(E-\mu) / k_{B} T\right]+1\right)^{-1}$. The factor 2 in the above equation is due to the holes in the valence band that contribute to $\chi$ in the same way as electrons in the con-

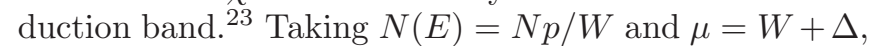
where $p$ is the number of states/cell, and $\mathrm{N}$ number of 


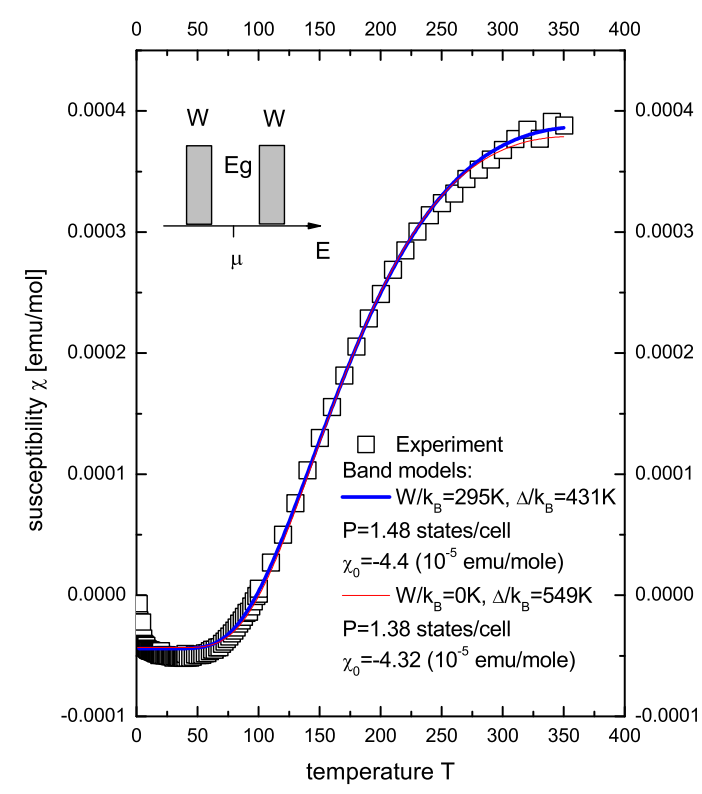

FIG. 5: Magnetic susceptibility of $\mathrm{FeSb}_{2}$ (data taken from Ref. 14). Note much smaller low temperature Curie tail than in FeSi. Solid and dashed lines represent fits using the model density of states shown in the inset.

unit cells, we obtain:

$\chi(T)=-2 \mu_{B}^{2} \frac{N p}{W} \frac{\exp (\beta \Delta)(1-\exp (\beta W))}{(1+\exp (\beta \Delta))(1+\exp (\beta(\Delta+W))}+\chi_{0}$

By fitting magnetic susceptibility data we obtain allowable values for parameters: $0 K<W / k_{B}<400 K$ and $850 \mathrm{~K}<\mathrm{Eg} / k_{B}<1100 \mathrm{~K}$, comparable to parameter values obtained for $\mathrm{FeSi}, \underline{3}$

The accumulation of states at the gap edges should in principle be observed in other thermodynamic properties, such as the thermal expansion $\alpha$ and the heat capacity $\mathrm{C}_{P}$. Unfortunately, there is no $M S b_{2}$ diamagnetic member with $\mathrm{M}=3 d$ transition metal element with the marcasite structure that we could use in order to extract the electronic contribution to $\alpha$ and $\mathrm{C}_{P}$. A similar difference has been obtained for FeSi using CoSi as a reference $\stackrel{3}{\underline{3}} \mathrm{CoSb}_{2}$ forms in a distorted monoclinic structure $P 2_{1} / c$ at ambient temperature and $\mathrm{NiSb}_{2}$, contrary to previously reported, is not diamagnetic ${ }^{24}$ From the low temperature specific heat (Fig. 6a) $C \sim B T^{3}$ where $B=\frac{12}{5} n R \pi^{4}\left(\frac{T}{\theta_{D}}\right)^{3}$, we estimate the Debye temperature as $\theta_{D}=(256 \pm 6) K$, a smaller value than previously reported $\left(\theta_{D}=380 \mathrm{~K}\right)$ based on vapor transport grown samples. Using the same range of parameters obtained from the magnetization fit, we have calculated spin state transition contribution to specific heat $\mathrm{C}_{P}=(\partial U / \partial T)$ with $\mathrm{U}$ given by the contributions from valence and conduction band:
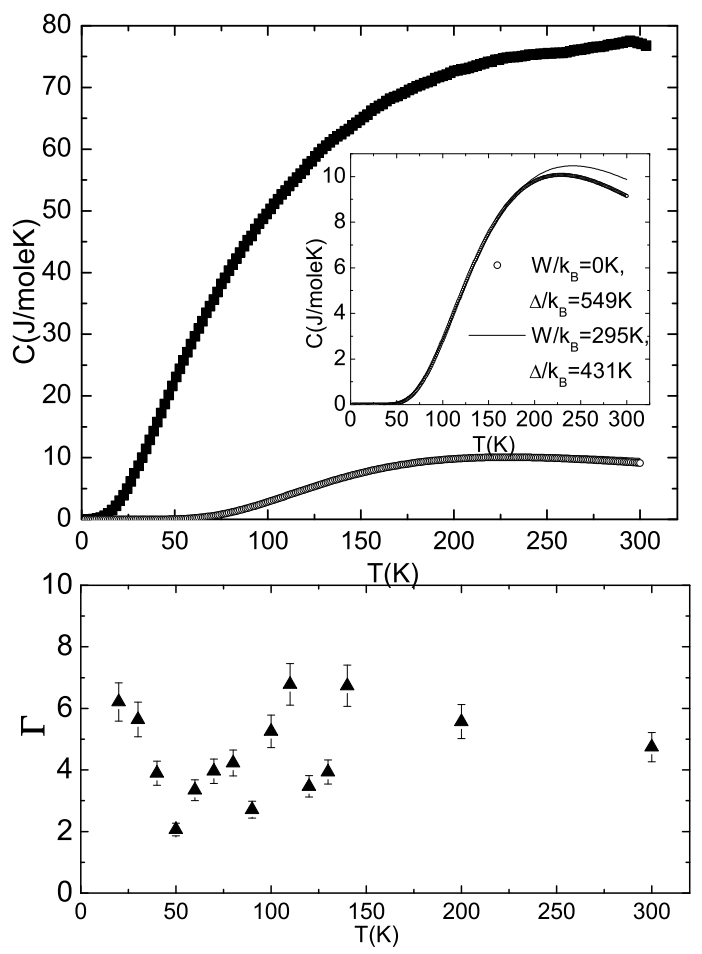

FIG. 6: (a) Heat capacity $\mathrm{C}_{P}$ of $\mathrm{FeSb}_{2}$. Inset shows contribution to heat capacity from the model density of states used to model magnetic susceptibility (see text). (b) Grüneisen parameter $\Gamma$ of $\mathrm{FeSb}_{2}$

$$
\begin{aligned}
U= & \int_{0}^{W} \frac{N p}{W} \frac{E d E}{\exp (\beta(E-W-\Delta))+1} \\
& +\int_{W+2 \Delta}^{2 W+2 \Delta} \frac{N p}{W} \frac{E d E}{\exp (\beta(E-W-\Delta))+1} .
\end{aligned}
$$

The results are shown in Fig. 6a where we see that phonon contribution dominates at $\mathrm{T} \sim \mathrm{T}_{\text {crossover }}$. From the measured specific heat (Fig. 6a) and the unit cell volume temperature dependance (Fig. 4) we estimate the Grüneisen parameter $\Gamma=3 B \alpha / C_{P}$ (Fig. 6b), where $\alpha=\frac{1}{3 V}\left(\frac{\partial V}{\partial P}\right)_{P}$ (Fig. 7) and B is the bulk modulus. The overall magnitude of the thermodynamic Grüneisen parameter $\Gamma$ is comparable to the one seen in $\mathrm{FeSi}^{25}$ We see that $\Gamma$ is weakly temperature dependant with a possible minimum developing around $T=50 \mathrm{~K}$. Since $\Gamma$ represents the measure of anharmonic effects in the lattice potential energy, our $\Gamma(T)$ indicates the overall agreement with the Grüneisen assumption $\Delta \omega / \omega=-\Gamma \Delta V / V$.

The $\alpha(T)$ for $\mathrm{FeSb}_{2}$ (Fig. 7) is different from the case of cubic or anisotropic metals, where $\alpha(T)$ follows the $\mathrm{C}_{P}$ decrease below room temperature in accord with the 


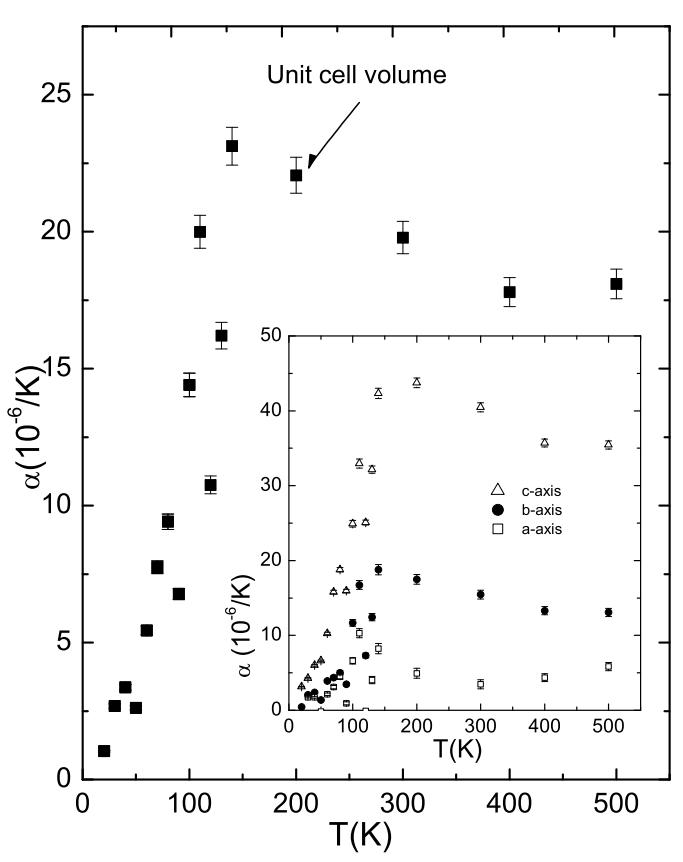

FIG. 7: Thermal expansion $\alpha=\frac{1}{V}\left(\frac{\partial V}{\partial P}\right)_{P}$ of the unit cell volume of $\mathrm{FeSb}_{2}$. Weak temperature dependance of the unit cell volume introduces some noise in thermal expansion data below 100K. Inset shows thermal expansion coefficient of the individual axes $\alpha=\frac{1}{l}\left(\frac{\partial l}{\partial P}\right)_{P}(\mathrm{l}=\mathrm{a}, \mathrm{b}, \mathrm{c})$

Debye function. Departures are expected for large variations of $\Gamma{ }^{26}$ The electronic contribution to the specific heat of a narrow gap semiconductor is expected to show a Schottky peak, as expected for a two level electronic system separated by gap $\mathrm{E}_{g}=2 \Delta$. If the degeneracies of levels separated by $\mathrm{E}_{g}$ are equal, we expect the peak at a temperature equivalent to $\Delta=2.4 T_{\text {peak }}, 27,28$ While we cannot extract the electronic contribution from the phonons in $\mathrm{C}_{P}$ (Fig. 6a shows that it is $\sim 10 \%$ of the measured value of $\mathrm{C}_{P}$ ), since the Grüneisen parameter $\Gamma=3 B \alpha / C_{P}$ remains roughly constant, the Schottky term should be reflected in the thermal conductivity as well. Notwithstanding that we were not able to extract the electronic contribution to $\alpha$, the data at Fig. 7 could be consistent with a broad peak forming at $\mathrm{T}_{\text {peak }} \sim(125$ $200) \mathrm{K}$, thus giving $\Delta / k_{B} \sim(300-480) K$. This might indicate that physically meaningful values of the gap are $850 K<E_{g} / k_{B}<960 K$, closer to the lower limit of those obtained from the fit of the magnetic susceptibility. Anisotropic thermal expansion coefficients of the the lattice constants $\alpha_{l}=\frac{1}{l}\left(\frac{\partial l}{\partial P}\right)_{P}(l=a, b, c)$ (Fig. 7 inset) show that the peak in the volume thermal expansion is dominated by the c-axis contribution $\alpha_{c}$, whereas we see little or no evidence for the peak formation for $\alpha_{a}$ or $\alpha_{b}$. Since the $\mathrm{d}_{x y}$ orbitals that could be involved in a spin state transition within the $t_{2 g}$ multiplet overlap along the c-axis of the crystal12 ${ }^{12}$, it is likely that the electronic states involved in the Schottky peak formation are in fact of $\mathrm{d}_{x y}$ origin. This agrees with Goodenough's picture of a band of itinerant states within the $t_{2 g}$ multiplet and observations of enhanced conductivity in paramagnetic region, $12,14,15$

In conclusion,we have shown that the experimental data for $\mathrm{FeSb}_{2}$ can be viewed as a thermally induced spin state transition within the Fe $t_{2 g}$ multiplet. A simple model with two peaks at the density of states at the gap edges can explain the thermally induced paramagnetic moment, as expected for Kondo Insulators. The magnetic properties correlate with the electronic conductivity in $\mathrm{FeSb}_{2}$ since the resistivity in the low spin diamagnetic state at $2 \mathrm{~K}$ is more than four orders of magnitude larger than in the paramagnetic high spin state at room temperature 14 This significant enhancement of the conductivity in the paramagnetic state agrees with Goodenough's hypothesis that thermal excitation populates $\Xi$ orbitals which have a substantial degree of covalent mixing and itineracy, as opposed to the more localized $\Lambda$ orbitals ${ }^{12}$ We see, however, that the magnetic susceptibility can be described by both a thermally induced Pauli susceptibility (Fig. 5) and a low spin to high spin transition $\stackrel{14}{14}$ Neutron scattering experiments would be very useful to quantify this and to correlate the phonon structure and frequency change with the thermally induced volume change. We conclude that the $\mathrm{FeSb}_{2}$ is a promising model system to study the applicability of the Kondo Insulator vs. the nearly itinerant magnetic semiconductor picture for $3 d$ intermetallic compounds, in addition to FeSi. This is further supported by preliminary optical spectroscopy measurements ${ }^{29}$

We thank Zachary Fisk, T. M. Rice and Igor Zaliznyak for useful communication. This work was carried out at the Brookhaven National Laboratory, which is operated for the U.S. Department of Energy by Brookhaven Science Associates (DE-Ac02-98CH10886) and at Ames Laboratory which is operated for the U.S. Department of Energy by the Iowa State University under Contract No. W-7405-82. This work was supported by the Office of Basic Energy Sciences of the U.S. Department of Energy and in part by the Serbian Ministry of Science and Technology through research grant 1899 .
${ }^{1}$ G. Aeppli and Z. Fisk, Comments Cond. Mat. Phys. 16, 155 (1992)

2 T. E. Mason, G. Aeppli, A. P. Ramirez, K. N. Clausen,
C. Broholm, N. Stücheli, E. Bucher, and T. T. M. Palstra, Phys. Rev. Lett 69, 490 (1992)

3 D. Mandrus, J. L. Sarrao, A. Migliori, J. D. Thompson 
TABLE I: FeSb $b_{2}$ variable temperature refinements. Space group Pnnm, Fe atom at $2 a$ site at (000), Sb atom at $4 g$ site at (xy0), sample contains $\sim 8$ wt.\% of Sb impurity. Other values are available upon request

\begin{tabular}{|c|c|c|c|c|c|c|c|c|}
\hline \hline $\mathrm{T}(\mathrm{K})$ & 20 & 100 & 150 & 200 & 250 & 300 & 400 & 500 \\
\hline \hline $\mathrm{a}(\AA)$ & $5.82117(6)$ & $5.82333(11)$ & $5.82480(8)$ & $5.82626(8)$ & $5.82755(8)$ & $5.82764(9)$ & $5.82957(8)$ & $5.83298(7)$ \\
\hline \hline $\mathrm{b}(\AA)$ & $6.50987(7)$ & $6.51336(12)$ & $6.51738(9)$ & $6.52304(9$ & $6.52911(9)$ & $6.53345(10)$ & $6.54221(9)$ & $6.55077(8)$ \\
\hline \hline $\mathrm{c}(\AA)$ & $3.16707(4)$ & $3.17035(6)$ & $3.17525(5)$ & $3.18218(5)$ & $3.18977(5)$ & $3.19630(5)$ & $3.20785(5)$ & $3.21926(4)$ \\
\hline \hline $\mathrm{V}\left(\AA^{3}\right)$ & $120.016(2)$ & $120.249(5)$ & $120.540(3)$ & $120.939(3)$ & $121.367(3)$ & $121.698(4)$ & $122.342(3)$ & $123.010(3)$ \\
\hline \hline $\mathrm{Sb}(\mathrm{x})$ & $0.1875(1)$ & $0.1872(2)$ & $0.1875(2)$ & $0.1882(2)$ & $0.1882(2)$ & $0.1887(2)$ & $0.1894(2)$ & $0.1901(2)$ \\
\hline \hline $\mathrm{Sb}(\mathrm{y})$ & $0.3554(1)$ & $0.3549(2)$ & $0.3550(2)$ & $0.3551(2)$ & $0.3558(2)$ & $0.3557(2)$ & $0.3564(2)$ & $0.3574(1)$ \\
\hline \hline$\chi^{2}$ & 6.740 & 9.606 & 8.822 & 7.671 & 8.842 & 9.145 & 8.186 & 7.671 \\
\hline \hline $\mathrm{Fe}-\mathrm{Sb} \times 2(\AA)$ & $2.5583(7)$ & $2.5558(9)$ & $2.5586(10)$ & $2.5628(10)$ & $2.5691(9)$ & $2.5708(10)$ & $2.5800(11)$ & $2.5904(9)$ \\
\hline \hline $\mathrm{Fe}-\mathrm{Sb} \times 4(\AA)$ & $2.5889(6)$ & $2.5931(8)$ & $2.5935(8)$ & $2.5934(8)$ & $2.5944(8)$ & $2.5951(9)$ & $2.5948(8)$ & $2.5945(7)$ \\
\hline \hline $\mathrm{Fe}-\mathrm{Sb}-\mathrm{Fe}(\circ)$ & $128.95(2)$ & $128.99(3)$ & $128.99(3)$ & $128.98(3)$ & $128.83(3)$ & $128.86(3)$ & $128.74(3)$ & $128.58(3)$ \\
\hline
\end{tabular}

TABLE II: FeSb 2 high pressure refinements. Space group $\mathrm{P}_{n n m}$, Fe atom at $2 a$ site at (000), Sb atom at $4 \mathrm{~g}$ site at (xy0), sample contains $\sim 8$ wt.\% of Sb impurity

\begin{tabular}{|c|c|c|c|c|c|c|c|c|}
\hline \hline $\mathrm{P}(\mathrm{GPa})$ & ambient & 0.79 & 1.98 & 2.91 & 3.75 & 4.15 & 5.93 & 7.14 \\
\hline \hline $\mathrm{a}(\AA)$ & $5.82788(9)$ & $5.8207(2)$ & $5.8042(2)$ & $5.7933(2)$ & $5.7843(2)$ & $5.7740(2)$ & $5.7583(2)$ & $5.7432(3)$ \\
\hline \hline $\mathrm{b}(\AA)$ & $6.53336(9)$ & $6.5198(2)$ & $6.4907(2)$ & $6.4733(2)$ & $6.4599(2)$ & $6.4456(2)$ & $6.4217(2)$ & $6.3986(3)$ \\
\hline \hline $\mathrm{c}(\AA)$ & $3.19626(5)$ & $3.1799(1)$ & $3.1570(1)$ & $3.1423(1)$ & $3.1312(2)$ & $3.1201(1)$ & $3.1012(1)$ & $3.0857(2)$ \\
\hline \hline $\mathrm{V}(\AA 3)$ & $121.699(4)$ & $120.676(6)$ & $118.933(6)$ & $117.843(5)$ & $117.001(6)$ & $116.118(4)$ & $114.677(5)$ & $113.394(6)$ \\
\hline \hline $\mathrm{Sb}(\mathrm{x})$ & $0.1887(1)$ & $0.1858(4)$ & $0.1854(4)$ & $0.1857(3$ & $0.1839(4)$ & $0.1850(3)$ & $0.1842(4)$ & $0.1842(4)$ \\
\hline \hline $\mathrm{Sb}(\mathrm{y})$ & $0.3561(1)$ & $0.3552(4)$ & $0.3537(3)$ & $0.3518(3)$ & $0.3527(3)$ & $0.3558(3)$ & $0.3585(4)$ & $0.3502(4)$ \\
\hline \hline$\chi^{2}$ & 6.681 & 2.625 & 2.394 & 6.126 & 4.001 & 19.91 & 22.06 & 12.89 \\
\hline \hline $\mathrm{Fe}-\mathrm{Sb} \times 2(\AA)$ & $2.5733(7)$ & $2.556(2$ & $2.535(2)$ & $2.519(2)$ & $2.515(2)$ & $2.530(2)$ & $2.535(2)$ & $2.478(2)$ \\
\hline \hline $\mathrm{Fe}-\mathrm{Sb} \times 4(\AA)$ & $2.5942(5)$ & $2.601(2)$ & $2.594(2)$ & $2.589(2)$ & $2.588(2)$ & $2.570(1)$ & $2.557(2)$ & $2.567(2)$ \\
\hline \hline $\mathrm{Fe}-\mathrm{Sb}-\mathrm{Fe}\left(^{\circ}\right)$ & $128.79(2)$ & $128.79(7)$ & $129.07(6)$ & $129.45(6)$ & $129.19(7)$ & $128.80(6)$ & $128.35(6)$ & $129.75(7)$ \\
\hline
\end{tabular}

and Z. Fisk, Phys. Rev. B 51, 4763 (1995)

4 D. N. Basov, F.S. Pierce, P. Volkov, S. J. Poon and T. Timusk, Phys. Rev. Lett 73, 1865 (1994)

5 Y. Nishino, M. Kato, S. Asano, K. Soda, M. Hayasaki and U. Mizutani, Phys. Rev. Lett 79, 1909 (1997)

6 Y. Takahashi and T. Moriya, J. Phys. Soc. Jpn. 46, 1451 (1979)

7 L. F. Mattheis and D. R. Hamann in Phys. Rev. B 47, 13114 (1993)

8 V. I. Anisimov, S. Y. Ezhov, I. S. Elfimov, I. V. Solovyev and T. M. Rice, Phys. Rev. Lett. 76, 1735 (1996)

9 V. I. Anisimov, J. Zaaneen and O. K. Anderson, Phys. Rev. B 44, 943 (1991)

10 F. Hulliger, Nature 198, 1081 (1963)

11 F. Hulliger in C.K. Jorgensen, J. B. Neilands, Sir Ronald S. Nyholm, D. Reinen and R. J. P. Williams (eds.), Structrure and Bonding, Vol. 4, 83 (1967)

12 J. B. Goodenough, J. Solid State Chem., 5144 (1972)

13 H. Holseth and A. Kjekshus, Acta Chemica Scandinavica 22, 3273 (1968), A. Kjekshus and T. Rakke, Acta Chemica Scandinavica, Series A 31A (7), 517 (1977), T. Rosenqvist,
Acta Metalurgica 4, 761 (1953)

14 C. Petrovic, J. W. Kim, S. L. Bud'ko, A. I. Goldman, P. C. Canfield, W. Choe and G. J. Miller, Phys. Rev. B 67, 155205 (2003)

15 A. K.L. Fan, G. H. Rosenthal, H. L. McKinzey and A. Wold, J. Solid State Chem., 5131 (1972)

16 T. Vogt et al., Phys. Rev. B 63, 220505

17 A. C. Larson and R. B. VonDreele, (Report LAUR 86-748, Los Alamos National Laboratory, New Mexico, 1986). B. H. Toby, J. Appl. Crystallogr. 34, 210 (2001).

18 Robert M. Hazen and Larry W. Finger in Comparative Crystal Chemistry, John Willey \& Sons 1982

19 G. Brostigen and A. Kjekshus, Acta Chem. Scand. 24, 1925 (1970)

20 H. Takizawa, T. Yamashita, K. Ueda and T. Endo. Phys. Stat. Sol. (b) 223, 35 (2001)

21 Angel, R.J. (2000) Equations of State. In R.M. Hazen, and R.T. Downs, Eds. Reviews in Mineralogy and Geochemistry: High-Temperature and High-Pressure Crystal Chemistry, 41, p. 35-58. The Mineralogical Society of America, Washington, DC 
${ }^{22}$ V. Jaccarino, G. K. Wertheim, J. H. Wernick, L. R. Walker and Sigurds Arajs, Phys. Rev 160, 476 (1967)

23 S. Paschen, E. Felder, M. A. Chernikov, L. Degiorgi, H. Schwer, H. R. Ott, D. P. Young, J. L. Sarrao and Z. Fisk, Phys. Rev. B 56, 12916 (1997)

${ }^{24}$ H. Holseth and A. Kjekshus, J. Less-Common Metals 16, 472 (1968)

${ }^{25}$ L. Vocadlo, K. S. Knight, G. D. Price and I. G. Wood, Phys. Chem. Minerals 29, 132 (2002)
26 T. H. K. Barron and G. K. White "Heat Capacity and Thermal Expansion at Low Temperatures", Plenum Publishing New York 1999

27 D. Mandrus, J. L. Sarrao, J. D. Thompson, M. F. Hundley, A. Migliori and Z. Fisk, Physica B 199-200, 471 (1994)

28 B. A. Aronzon, G. Galeczki, G. Nimtz, Phil. Mag. B 67, $847(1993)$

29 L. de Giorgi, private communication 